\title{
FAMILIAS NO SAGRADAS: MARIOLA PIETRAK, HACIA LA POS / FAMILIA. REPRESENTACIONES DE LA FAMILIA EN SIETE AUTORAS ARGENTINAS (1981-2013). SEVILLA: PADILLA LIBROS, 2018
}

El objeto de estudio del libro de Mariola Pietrak Hacia la pos / familia. Representaciones de la familia en siete autoras argentinas (1981-2013) es la representación de la familia en la narrativa argentina actual. En su introducción la autora hace hincapié en la complejidad del tema, solo aparentemente sencillo. El análisis -como señala- tendrá un carácter interdisciplinario, porque la familia es un fenómeno multidimensional, situado en la encrucijada de varias: biológica, psicológica, afectiva, social, económica o política. Es más, estas perspectivas se mezclan entre sí, se superponen, se transforman imperceptiblemente unas en otras.

El tema de la familia adquiere connotaciones adicionales, aún más complejas, en el contexto local argentino. Esto se evidencia por la intensificación de su representación en la prosa nacional, tanto la de hace unas décadas, como la actual. Sin duda, en este estado de cosas influyeron los turbulentos acontecimientos históricos y sus consecuencias sociales y políticas que atormentaban a la patria de Borges. Un ejemplo paradigmático de la interrelación entre las cuestiones familiares y estatales lo representa la famosa organización de Madres de Plaza de Mayo, que desde hace más de 40 años reúne a mujeres cuyos hijos "desaparecieron" en la llamada "guerra sucia" durante la dictadura militar entre los años 1976 y 1983.

Las familias argentinas están unidas en la lucha por la verdad y la justicia violadas por el terror de Estado de la pasada dictadura. También muestran solidaridad frente a los acontecimientos políticos contemporáneos. Uno de ellos es la gran crisis económica, comúnmente conocida como corralito, que afectó a la Argentina a principios de este siglo. El colapso de la economía argentina, resultado de decisiones irresponsables del gobierno, empeoró significativamente las condiciones de vida, especialmente de los más desfavorecidos, al restringir los derechos civiles fundamentales, como el acceso sin trabas a la educación, la atención de la salud o las oportunidades profesionales. Sin embargo, la creciente importancia de la categoría familiar no sólo concierne a la Argentina, sino que es un fenómeno universal, global. Actualmente, en la mayoría de los países occidentales se están llevando a cabo negociaciones para la redefinición de la célula social más pequeña, en la que participan los actores enfrentados: el aparato estatal, la Iglesia, los movimientos feministas o LGBT ${ }^{1}$.

\footnotetext{
${ }^{1}$ En los últimos años se han publicado varios textos sobre la representación literaria de la familia, entre los que pueden mencionarse: Pérez Heredia, Alexander (2003) "Familia e identidad en la novísima literatura cubana de los ochenta"; Domínguez, Nora (2004) "Familias literarias: Visión adolescente y poder político en la narrativa de Beatriz Guido"; Margarida Ramos, Ana (et al., 2013) La familia en la
} 
La autora, por un lado, confronta al lector con una maraña de problemas enraizados en torno al tema central, que presenta desde diferentes perspectivas y puntos de vista; por otro, mantiene un orden meticuloso a la hora de definir los aspectos que serán de particular interés para su análisis. Los objetivos del estudio se presentan de forma clara y sugerente. El primer capítulo del libro, titulado "Familia, sociedad, literatura", está dedicado a la presentación de los fundamentos de la metodología de la investigación, orientada principalmente en torno a la teoría sociocrítica. La investigadora expone los supuestos básicos de esta concepción, explicando, entre otras cosas, en qué se diferencia de la perspectiva sociológica. Es una aclaración valiosa, puesto que a veces, también en los trabajos científicos, se producen malentendidos o inexactitudes en este campo. Así, las afirmaciones del tipo "el objeto del estudio no son las familias reales, sino sus representaciones literarias" (p. 17) o incluso más enfáticas: "representación y realidad no son la misma cosa" (p. 17), no parecen en absoluto redundantes; por el contrario, subrayan el carácter estrictamente téorico-literario del análisis, que a veces en este tipo de obras se relega a segundo plano.

La parte teórica del libro, repleta de concepciones filosóficas sobre la ideología, disciplina y poder, en la que se inscribe el fenómeno de la familia, se caracteriza por una verdadera erudición. Mariola Pietrak utiliza hábilmente los conceptos propuestos por Michel Foucault, Luis Althauser o Pierre Bourdieu, demostrando que está equivocado quien piensa que la familia es un ente "natural", universal, desprovisto de enredos ideológicos, un fenómeno que pertenece a la esfera privada. La investigadora enfatiza su función socializadora: el sumergir al individuo en el sistema de relaciones sociales de dominación y subordinación. La familia es una institución fundamental responsable de la normalización del individuo, que opera sobre el principio de dispositivo realizado a través de los mecanismos de la disciplina.

Las prácticas de socialización que se realizan a través de la institución familiar, que es a la vez su resultado, se efectúan, entre otros, en el ámbito lingüístico, a través de prácticas discursivas. El primer capítulo del libro se centra en el aspecto lingüístico de la constitución de la subjetividad. Los discursos sociales y los textos literarios están estrechamente relacionados e interactúan entre sí. La estudiosa se enfoca en explicar cómo los elementos ideológicos se insertan en los textos literarios. Para ello, se basa principalmente en la concepción de una filóloga polaca, especializada en teoría sociocrítica, Pierrette Malcuzynski. ${ }^{2}$ La autora utiliza pues las herramientas metodológicas tomadas del aparato conceptual propio de la teoría sociocrítica, como el monitoring, el ideologema o el ideosema. Desafortunadamente, la erudición de Pietrak a veces va en contra de la accesibilidad y el placer de la lectura. La acumulación de términos científicos sofisticados y definiciones complejas, que a menudo se explican a través de interjecciones con citas de otros investigadores, dificulta al lector la comprensión de algunos enunciados. Oraciones del tipo:

\footnotetext{
literatura infantil y juvenil; Maradei, Guadalupe (2016) "Cuerpos que insisten: familia, matrimonio y maternidad en la literatura argentina de la última década"; Sanabria, Carolina (2016) "La representación del espacio familiar como ominoso en la literatura costarricense: de La ruta de su evasión a Larga noche hacia mi madre".

${ }^{2}$ Para saber más sobre la teoría de Pierrette Malcuzynski, véase el número especial de Itinerarios. Revista de estudios lingüisticos, literarios, históricos y antropológicos, 2009, n 10.
} 
Teniendo en cuenta que el sujeto psíquico se define como la suma de experiencias integradas en su paso por distintos campos y estratos sociales en diferentes momentos de la vida, como la suma de interferencias de las memorias colectivas de varios grupos que conforma (Cros 2015, 47, Lavabre 2007, Halsbwachs 2004, Hirsch 1997), su mediación en los textos producidos -en estas petites histoires a las que hace referencia el título original- da cuenta de la heteroglosia del capo discursivo desde el interior mismo de los discursos minoritarios informando no solo de las tensiones sociales que subyacen al trauma y permanecen en el "pretérito presente" de la narración, tensiones que en el presente oponen memoria a historia, trauma personal a trauma colectivo, testimonio a ficción... (Pietrak, 2018, p. 205)

son sencillamente demasiado largas y en exceso complejas. Por supuesto, se trata de una cuestión más bien secundaria, que no disminuye el valor de esta importante publicación.

Al capítulo teórico le sigue la parte analítica de la monografía. El objeto del análisis lo constituye la selección de textos de siete escritoras argentinas: Marta Traba, Matilde Sánchez, Laura Alcoba, Raquel Robles, Ángela Urondo Raboy, Patricia Suárez y Florencia Abbate. Ninguna de las novelas en cuestión ha sido publicada en nuestro país, por lo que las autoras cuyas obras han sido analizadas no son conocidas por el lector polaco corriente. A pesar de ello, o quizás paradójicamente debido a ello, la monografía podría traducirse al polaco. La literatura latinoamericana contemporánea, especialmente la femenina, no es particularmente difundida en nuestro país, y, en consecuencia, se echa en falta también la literatura crítica que se dedique a ella.

La más temprana de las novelas analizadas fue publicada en el año 1981, la más reciente es de 2013. El enfoque diacrónico nos permite observar la evolución de la familia, influenciada por las nuevas circunstancias sociales y políticas. De ahí el título "pos/familia", un término acuñado por Elisabeth Beck-Gernsheim ${ }^{3}$ para referirse a las formas familiares contemporáneas y en transformación, que difieren significativamente de su versión tradicional. ${ }^{4}$ Mariola Pietrak avanza con fluidez hacia el estudio de los sucesivos textos literarios, sin precederlo con excesiva información biográfica de escritoras, lo que es una práctica utilizada a menudo en publicaciones similares y cuyo resultado no es raro que sea una suma de fragmentos semejantes a notas de la Wikipedia, lo que acaba perturbando la dinámica del análisis

\footnotetext{
${ }^{3}$ Socióloga y académica alemana; sus principales intereses de investigación son los cambios sociales relacionados con la familia. El concepto de la posfamilia lo describe por primera vez en su libro Reinventing the family: in search of new lifestyles (2002), Nueva Jersey: Wiley-Blackwell.

${ }^{4}$ Tras ser preguntada en una entrevista sobre la significación del concepto de la posfamilia, BeckGernsheim explica: "Esto no quiere decir que la familia estándar haya desaparecido. Sin embargo, hoy en día existe una diversidad mucho mayor de formas y arreglos familiares y sobre todo: el modelo estándar ha perdido su fuerza normativa. En las últimas décadas se han producido cambios, tanto en el comportamiento familiar, como en la comprensión normativa de la célula más pequeña de la sociedad y ahora coexisten diferentes formas de familia, cada una de las cuales reivindica la igual consideración. Este es, en efecto, el punto crucial: la forma familiar tradicional ha perdido su antigua posición de monopolio; en su lugar, ahora existen ideas de normalidad diferentes en competencia y cada vez más formas se consideran opciones legítimas. No es sólo el aumento y el incremento de las «desviaciones» lo que caracteriza nuestra situación actual. Aún más importante es que las formas anteriormente «desviadas» se hayan convertido en una de las muchas variedades de lo normal y por lo tanto son socialmente aceptadas" (Ravn, T., Sørensen, M., 2013). Traducción propia de inglés: W.S.
} 
principal. No obstante, una información apropiada y sintética sobre las escritoras comentadas se puede encontrar al final del libro.

La interpretación literaria de Pietrak es fascinante. Las descripciones de los elementos de la trama están entrelazadas armoniosamente con comentarios críticos y motivos teóricos. Particular relevancia merece el carácter multidimensional de los estudios. De acuerdo con lo anunciado en la introducción, la autora examina el fenómeno de la familia desde diferentes perspectivas, combinando hábilmente varios hilos interpretativos. La figura de la familia introduce en la investigación las siguientes categorías literarias, orientadas en su campo semántico: post-memoria, hogar, archivo, nación, identidad. Nos balanceamos todo el tiempo en la frontera entre lo natural, lo privado y lo social, lo público. La familia se deconstruye, se desintegra, pierde consistencia, pero pronto se reconstituye, se une de nuevo, para sobrevivir en una forma transformada y diferente, a pesar de todo. 


\section{BIBLIOGRAFÍA}

Beck-Gernsheim, E. (2002). Reinventing the family: in search of new lifestyles. Nueva Jersey: Wiley-Blackwell.

Domínguez, N. (2004). Familias literarias: Visión adolescente y poder político en la narrativa de Beatriz Guido. Revista Iberoamericana, LXX, 206, 225-235.

Flisek, A., Moszczyńska-Dürst, K. (coords.) (2009). Homenaje a M.-Pierrette Malcuzynski. Itinerarios. Revista de estudios lingüisticos, literarios, históricos y antropológicos, 10.

Maradei, G. (2016). Cuerpos que insisten: familia, matrimonio y maternidad en la literatura argentina de la última década. Chasqui. Revista de literatura latinoamericana, 45.1, 246-263.

Margarida Ramos, A. et al. (2013). La familia en la literatura infantil y juvenil. Vigo-Braga: Asociación Literaria Nedro.

Pérez Heredia, A. (2003). Familia e identidad en la novísima literatura cubana de los ochenta. Revista Universidad de La Habana, 257, 20-32.

Ravn, T., Sørensen, M. (2013). Interview with Elisabeth Beck-Gernsheim on Individualization. Theory, Culture and Society. Consultado el 16 de octubre de 2019 https://www.theo ryculturesociety.org.

Sanabria, C. (2016). La representación del espacio familiar como ominoso en la literatura costarricense: de La ruta de su evasión a Larga noche hacia mi madre. Filología y Lingüistica, 42, 117-127. 